\title{
Evaluación Rápida de Potencial de Riesgo Genotóxico (ERPRG) asociada a metales pesados en agua potable: Caso de estudio Zimapán, Hidalgo, México. Rapid Assessment of Genotoxic Risk Potential (RAGRP) associated with heavy metals in drinking water: A case study from Zimapán, Hidalgo, México.
}

\author{
M. A. Sánchez-Olivares (iD a , J. C. Gaytán-Oyarzun (iD a*, F. Prieto-García (iD b y R. B. E. Cabrera-Cruz (D) c \\ a Área Académica de Biología, Universidad Autónoma del Estado de Hidalgo. Carretera Pachuca-Tulancingo, Km 4-5- Pachuca. C.P. 42076. Mineral de la \\ Reforma, Hidalgo, México. \\ ${ }^{b}$ Área Académica de Química, Universidad Autónoma del Estado de Hidalgo. Carretera Pachuca-Tulancingo, Km 4-5- Pachuca. C.P. 42076. Mineral de la \\ Reforma, Hidalgo, México. \\ 'Facultad de Ingeniería “Arturo Narro Siller”. Universidad Autónoma de Tamaulipas. Centro Universitario Sur, C.P. 89100. Tampico, Tamaulipas. México.
}

\begin{abstract}
Resumen
Se presenta un método de análisis rápido para la evaluación de potencial de riesgo genotóxico asociado a metales pesados presentes en agua potable. Este método se basa en el análisis de las principales características fisicoquímicas, cinética ambiental, biodisponibilidad, efectos biológicos reportados y/o potenciales que puedan incrementar la probabilidad de manifestar un efecto adverso. Se planteó un caso de estudio en Zimapán Hidalgo, debido a que existen evidencias de la presencia de metales pesados de interés toxicológico en el agua potable. Los resultados de la aplicación de la metodología ERPRG muestran que el cromo, cadmio, arsénico y plomo se encuentran en la categoría de potencial de riesgo alto; mientras que el mercurio en la categoría de riesgo intermedio. ERPRG, es una herramienta que permite identificar situaciones de potencial de riesgo biológico y ambiental, basada en el análisis sistemático de reportes bibliográficos y estudios previos que permiten determinar cualitativamente el impacto de variables ambientales, biológicas y situacionales de manera clara, rápida y resumida.
\end{abstract}

Palabras Clave:

Evaluación de riesgo, ecotoxicología, metales pesados, riesgo genotóxico.

\begin{abstract}
A rapid analysis method is presented for the assessment of genotoxic risk potential associated with heavy metals present in drinking water. The method is based on analysing the main physical and chemical characteristics, environmental kinetics, bioavailability, reported and/or potential biological effects that may increase the probability of an adverse effect. A case study was conducted in Zimapan, Hidalgo, because there is evidence of the presence of heavy metals of toxicological interest in the drinking water. The results of the application of the RAGRP show that chromium, cadmium and arsenic and lead, are in the potential high risk category; while mercury is in the intermediate potential risk category. RAGRP, is a useful tool to identify situations of potential biological and environmental risk based on a systematic analysis of bibliographic reports and previous studies to qualitatively determine the impact of environmental, biological and situational variables in a clear, rapid and summarized manner.
\end{abstract}

Keywords:

Risk assessment, ecotoxicology, heavy metals, genotoxic risk.

\section{Introducción}

La contaminación ambiental es un problema mundial, donde la mayoría de los contaminantes de mayor relevancia en los sistemas acuáticos son los metales pesados. Por su elevada toxicidad, el impacto ocasionado en la salud por exposición prolongada y por su bioacumulación, resulta alarmante. Dependiendo de la especie química de cada tipo de metal o metaloide, se producen afecciones que van desde daños en órganos vitales hasta desarrollos cancerígenos (Combariza, 2009; Nava-Ruíz and MéndezArmenta, 2011). Comprender la distribución y comportamiento de estos contaminantes es crítico para la gestión ambiental e identificar el riesgo potencial que presentan a los sectores expuestos (Liu et al., 2006). Sin embargo, el establecimiento de

\footnotetext{
*Autor para la correspondencia: jcgaytan@uaeh.edu.mx García), rcabreracruz@uat.edu.mx (Rene Bernardo Elías Cabrera-Cruz).

Correo electrónico: sanchezma8@gmail.com (Marco Antonio Sánchez-Olivares), jcgaytan@uaeh.edu.mx (Juan Carlos Gaytán-Oyarzun), prietog@uaeh.edu.mx (Francisco Prieto- 
laboratorios de análisis y la aplicación de técnicas aceptables bajo normativa internacional es una tarea relativamente cara. Esta es una de las principales limitantes que ocasiona la falta de programas de monitoreo en países en vías de desarrollo como en Latinoamérica (Díaz-Barriga, 1999).

En las últimas décadas, se han desarrollado y validado métodos de evaluación de riesgos que permiten valorar el peligro que una sustancia o compuesto pueda ocasionar al ambiente y sus sectores expuestos. Estos métodos, son procesos sistemáticos que consisten en recopilar, evaluar y documentar información con el fin de determinar un nivel de riesgo. La información proporcionada puede ser utilizada para regular el uso de una sustancia o según las consideraciones políticas, sociales, económicas y técnicas en el proceso de gestión de riesgos. Los datos requeridos para la evaluación de riesgos en relación a la salud humana pueden clasificarse como datos sobre la identidad de la sustancia o compuesto, propiedades fisicoquímicas, toxicológicas y datos sobre la exposición a la misma (PérezRodrigo et al., 2015).

En México, por desconocimiento, los estudios de evaluación de riesgos son escasos y se han basado en métodos detallados que requieren de mucho tiempo para obtener resultados confiables, esto plantea la necesidad de desarrollar metodologías que permitan lograr resultados en corto tiempo (Zepeda et al., 2002). Es así, como diversos autores han desarrollado métodos de evaluación rápida que apoyen la toma de decisiones basados en información contextual de manera rápida, clara y eficaz.

Entre las alternativas existentes para llevar a cabo la toma de decisiones, se destaca la Evaluación Rápida de Fuentes de Contaminación Ambiental (ERFCA) (en aire, agua y suelos) (WHO, 1982); la Evaluación Rural Rápida (ERR) (Chambers, 1983); el Método de Evaluación del Riesgo de Extinción de las Especies Silvestres en México (Tambutti et al., 2001); la Evaluación Rápida de Biodiversidad para Estimar Prioridad Biológica (ERBio) (Gaytán et al., 2012); la Evaluación Rápida de Potenciales de Riesgo, aplicada a compuestos orgánicos persistentes (COPs) (Quiterio-Pérez, 2012; Gaytán y López, 2019) y la Evaluación de Potencial de Riesgos Ambientales y Efectos a la Salud (EPRAS) aplicada a antiinflamatorios no esteroideos (AINEs) (Rodríguez-Anaya et al., 2014); todos estos métodos sustentan la toma de decisiones de una manera imparcial, de una forma rápida basada en un análisis dirigido de datos publicados, cada una de ellas teniendo en común el no presentar una fase experimental en su ejecución, por lo tanto recaen en un conjunto de métodos denominados "Evaluación Rápida".

El objetivo del presente trabajo es proporcionar un método de análisis rápido para la toma de decisiones, sustentado en estudios previos de química analítica y/o reportes bibliográficos de nivel técnico científico a profesional que permitan evidenciar la presencia de metales pesados en el sitio de estudio y la probabilidad de existencia de posibles efectos genotóxicos asociados a la exposición.

\section{Materiales y Métodos}

Para evaluar el potencial de riesgo de metales pesados y metaloides, se requiere de una técnica de evaluación rápida para evaluar el potencial de riesgo asociado que se denominará "Evaluación Rápida de Potenciales de Riesgo Genotóxico (ERPRG)", la cual se basa en tres criterios independientes que pueden afectar la posibilidad de que un riesgo se manifieste en una población a través de un efecto negativo: 1) Variables ambientales y situacionales; 2) Variables individuo-específicas y 3) Variables fisicoquímicas del compuesto químico. El presente método, consiste en una revisión bibliográfica sistemática y exhaustiva, partiendo del supuesto de la existencia del compuesto a evaluar en el sitio de estudio y de un análisis de causa y efecto enfocado al peligro, exposición y efecto potencial de la exposición al compuesto xenobiótico. Este método, se implementó en el sitio de estudio ubicado en el municipio de Zimapán, perteneciente al estado de Hidalgo, México, en este sitio, se cuenta con reportes de la presencia de metales pesados y metaloides en el agua potable, todos ellos de interés toxicológico, debido a reportes previos de efectos tóxicos y secundarios reportados con potencial carcinógeno, mutagénicos y teratogénico; de acuerdo a su origen, cada uno de ellos se encuentra de manera natural en forma mineral, con base a las propiedades fisicoquímicas ambientales y a las características propias de cada compuesto, todos ellos presentan cinética ambiental y alta probabilidad de ser biodisponibles, así como bioacumulables en sistemas biológicos.

\subsection{Recopilación de datos}

Para llevar a cabo esta fase, se procedió a la recopilación de datos, esta fue a partir de reportes bibliográficos desde un nivel técnico científico a profesional, de manera sistemática y exhaustiva, donde no sea requerida una fase experimental para la obtención de dicha información; en esta recopilación se identificaron similitudes y diferencias entre los compuestos a evaluar (As, Cr, Cd, Pb y Hg), mismas que con un análisis de causa y efecto fue posible identificar las principales variables que podrían incrementar el potencial de riesgo.

\subsection{Método de análisis}

Para evaluar los aspectos anteriores se designaron 15 indicadores ambientales y/o biológicos, cada una de ellas con 3 a 5 variables a contemplar, con valores numéricos asignados para su fácil interpretación en una matriz comparativa de dos vías. La puntuación fue de 1 a 5 , donde 5 representa la mayor probabilidad. Si los criterios establecidos están asociados a un cálculo matemático o clasificación previa son preferentes para evitar ambigüedades en su interpretación; en caso de no existir un dato o referencia bibliográfica se le asignó un valor de 1 por el simple hecho de probabilidad de existencia e incertidumbre. La suma total de los valores asignados correspondió a aquel compuesto con una mayor capacidad de ejercer daño en la población (Gaytán et al., 2012; Quiterio-Pérez, 2012; Rodríguez-Anaya et al., 2014; Gaytán y López, 2019; Gaytán-Oyarzun et al., 2020).

El siguiente instrumento de evaluación es parte de esta metodología de evaluación rápida, que permita discernir las diferencias entre factores ambientales, situacionales, del sector expuesto, así como propias de los compuestos químicos o elementos a evaluar, de manera individual o por interrelaciones entre cada una de ellas (peligro, exposición y efecto) (Fig. 1).

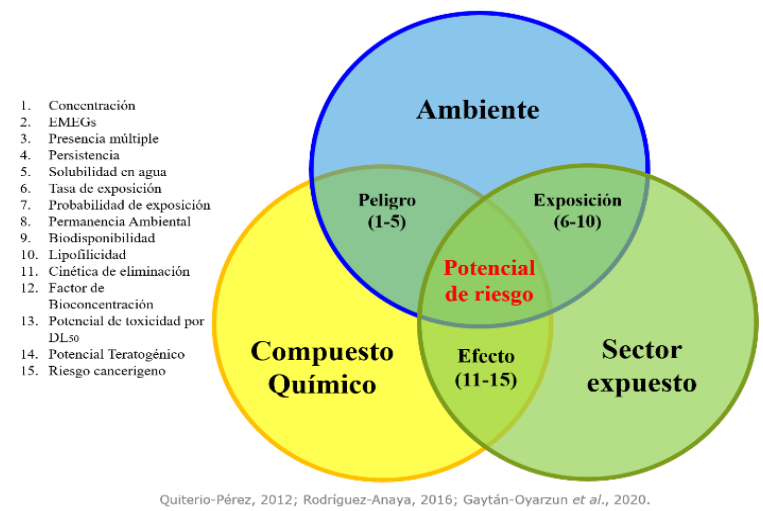

Figura 1. Diagrama de Venn de Potencial de Riesgo construido con indicadores exógenos (Gaytán-Oyarzun et al., 2020). 


\subsection{Análisis de causa y efecto}

$>$ Identificación y caracterización del peligro: Consiste en obtener una descripción cualitativa o cuantitativa de las propiedades inherentes de los compuestos xenobióticos que tienen el potencial de causar efectos adversos (OMS, 2017), este puede ser determinado a través de 5 indicadores relacionados con características del comportamiento fisicoquímico del contaminante al estar presente en el medio a evaluar: concentración, contaminante critico en valores EMEG (Guía de Evaluación para Medios Ambientales, EMEG por sus siglas en inglés), presencia múltiple, persistencia y solubilidad en agua.

1) Peligro con base en su concentración: Este rubro se refiere a la probabilidad de que la concentración de metales pesados y metaloides reportados en el agua de consumo. Para el cálculo de la peligrosidad mediante el cociente de peligro (González-Mille et al., 2010), se considera la concentración de exposición (reportada) sobre la concentración de referencia establecida como límite máximo permitido (LMP) por la NOM-127-SSA1-2017, en agua para uso y consumo humano. Si el resultado del cálculo del cociente se encuentra en o por debajo no representa una peligrosidad ambiental; mientras que, si la concentración de referencia o si el valor crece progresivamente sobre uno, indica una mayor peligrosidad ambiental expresados en ordenes de magnitud (CEPIS/OPS, 2005).

$$
\mathrm{CP}=\mathrm{Ce} / \mathrm{Cr}
$$

Dónde: (CP) Cociente de peligro; (Ce) Concentración de exposición (reportada); (Cr) Concentración de referencia (LMP, NOM-127-SSA1-2017).

a) $\mathrm{CP}(\geq 3)$. Peligrosidad alta $=5$

b) $\mathrm{CP}(1-3)$. Peligrosidad media-alta $=4$

c) $\mathrm{CP}$ (1). Peligrosidad media $=3$

d) $\mathrm{CP}(\leq 1)$. Peligrosidad baja $=2$

e) Desconocido $=1$

2) Peligro con base la estimación del contaminante critico en valores EMEG: Este rubro se refiere a la concentración ambiental estimada a la que los seres humanos pueden estar expuestos durante un periodo de tiempo específico sin experimentar efectos adversos para la salud (ATSDR, 2005); se basa el cálculo de los valores EMEG obtenida para el agua potable utilizada en los hogares $\left(E^{2} \mathrm{EG}_{\mathrm{w}}\right)$. El cálculo de la EMEG se obtiene multiplicando la dosis de referencia (RfD) de la base de datos IRIS de la EPA (2005) o el Nivel de Riesgo Mínimo (MRL) de la ATSDR (2004) por el peso corporal y dividiendo el producto entre la tasa de ingestión diaria de agua. El contaminante cuya concentración en el ambiente supere la concentración ambiental estimada, se considera un contaminante critico lo cual aumenta su peligrosidad (Díaz-Barriga, 1999).

$$
E M E G w=\frac{R f D \text { ó } M R L(m g / k g / \text { dia }) \times P C(K g)}{T I(L / \text { día })}
$$

Dónde: $\left(\mathrm{EMEG}_{\mathrm{w}}\right)$ Guía de Evaluación para medios ambientales en agua (mg/L); (RfD o MRL) Dosis de Referencia o Dosis de Riesgo Mínimo (Banco de datos IRIS o ASTDR); (PC) Peso corporal 70 $\mathrm{kg} /$ adulto, $16 \mathrm{~kg} / \mathrm{niños}$ (1-6 años), $10 \mathrm{~kg} /$ infantes (6-11 meses); (TI) Tasa de Ingestión diaria de agua $=2$ L/adultos.

a) Peligrosidad alta. La concentración ambiental supera el valor de $\mathrm{EMEG}_{\mathrm{w}}=5$

b) Peligrosidad media. La concentración ambiental es igual al valor de $\mathrm{EMEG}_{\mathrm{w}}=4$ c) Peligrosidad baja. La concentración ambiental se encuentra por debajo del valor de $\mathrm{EMEG}_{\mathrm{w}}=3$

d) Valor de EMEG $_{\mathrm{w}}$ desconocido $=1$

3) Peligro con base en su presencia múltiple: Este rubro permite determinar la peligrosidad de los compuestos xenobióticos, a través del incremento de la presencia del compuesto en múltiples matrices bióticas o abióticas (agua, aire y suelo), lo cual se relaciona con la cinética ambiental del contaminante (MartinOlmedo et al., 2016).

a) En diferentes medios abióticos (agua, aire y suelo) y bióticos $=5$

b) En dos o más medios abióticos (agua, aire y/o suelo) $=4$

c) Un medio abiótico (agua, aire y/o suelo) $=3$

d) Desconocido $=1$

4) Peligro basado en su persistencia a través de su vida media en el ambiente: Se refiere al tiempo para que un compuesto químico alcance la mitad de su concentración en el ambiente, ya que a mayor temporalidad mayor peligrosidad ambiental (Ramírez y Lacasaña, 2001) (Tabla 1).

Tabla 1. Clasificación de metales y metaloides basada en su vida media (modificada de Ramírez y Lacasaña, 2001).

\begin{tabular}{ccc}
\hline Persistencia & Vida media & Ejemplos \\
\hline Permanente & $\begin{array}{c}\text { Indefinidamente } \\
\text { Varios meses a } 50\end{array}$ & $\mathrm{Cd}, \mathrm{Pb}$ \\
$\begin{array}{c}\text { años } \\
\text { Modersistente } \\
\text { persistentes }\end{array}$ & $\begin{array}{c}1 \text { a } 18 \text { meses } \\
\text { De días a 12 } \\
\text { semanas }\end{array}$ & $\mathrm{Hg}$ \\
\hline
\end{tabular}

a) Permanentes (Indefinidamente) $=5$

b) Persistentes (De varios meses a 50 años) $=4$

c) Moderadamente persistentes (de 1 a 18 meses) $=3$

d) No persistentes (de días hasta 12 semanas) $=2$

e) Persistencia desconocida $=1$

5) Peligro con base a su solubilidad en agua: Este rubro se refiere a la capacidad de un compuesto xenobiótico de disolverse en otra sustancia, para calcular la solubilidad de los metales en el agua, se emplea la constante de equilibrio de solubilidad (pKs) (GarritzRuiz et al., 2005). Entendiéndose que valores de pKs mayores, presentan mayor solubilidad en agua, lo que indica mayor peligrosidad ambiental y favorece su cinética ambiental.

a) Valor de pKs $(\geq 20)$. Altamente soluble $=5$

b) Valor de pKs (10-19). Medianamente soluble $=4$

c) Valor de pKs $(\leq 9)$. Baja solubilidad $=3$

d) Valor de $\mathrm{pKs}$ desconocido $=1$

$>$ Evaluación de la exposición: Se emplea la evaluación de exposición para determinar, el contacto directo o indirecto del sector expuesto con los compuestos xenobióticos potencialmente peligrosos (OMS, 2017), esta puede ser evaluada a través de 5 indicadores relacionados con una serie de factores del ambiente en las que se ve involucrado el sector expuesto: tasa de exposición, probabilidad de exposición por sector expuesto, permanencia ambiental y su relación con el periodo de vida del sector expuesto, biodisponibilidad y lipofilicidad.

1) Exposición basada en la Tasa de exposición (TE): Este rubro se refiere a la estimación de la concentración del compuesto químico en el medio de exposición durante una duración determinada. La tasa de exposición se calcula como la 
concentración de un compuesto químico en un medio de exposición multiplicada por la tasa en que una persona ingiere dicho medio, dividido entre el peso corporal (ATSDR, 2005).

$$
T E(m g / k g / \text { día })=\frac{(\text { Conc. })(T I)(F E)}{P C}
$$

Dónde: (TE) Tasa de exposición; (Conc) concentración del contaminante en el medio ambiental (mg/L); (TI) Tasa de ingesta de agua (mg/L/día), asumiendo consumo por adultos de 2 litros/adulto; (PC) peso corporal $70 \mathrm{~kg} /$ adulto, $16 \mathrm{~kg} / \mathrm{niños}$ (1-6 años), $10 \mathrm{~kg} /$ infantes (6-11 meses); (FE) Factor de exposición (días/año); al estimar exposiciones agudas, a corto plazo o en situaciones en las que las exposiciones sean diarias a lo largo del tiempo, se asume un factor de exposición con valor de 1 (ATSDR, 2005). Los datos pueden provenir de la literatura científica y del estudio efectuado en el sitio. Entendiéndose que una alta tasa de exposición se asocia con una mayor exposición.

a) Probabilidad alta Tasa de Exposición $\left(10^{-3} \mathrm{mg} / \mathrm{kg} /\right.$ día $)=5$

b) Probabilidad media-alta Tasa de Exposición $\left(10^{-4}\right.$ $\mathrm{mg} / \mathrm{kg} / \mathrm{día})=4$

c) Probabilidad media Tasa de Exposición $\left(10^{-5} \mathrm{mg} / \mathrm{kg} / \mathrm{día}\right)=3$

d) Probabilidad baja Tasa de Exposición $\left(10^{-6} \mathrm{mg} / \mathrm{kg} /\right.$ día $)=2$

e) Probabilidad de exposición desconocido $=1$

2) Probabilidad de exposición para el sector poblacional: Este rubro determina la probabilidad de exposición en la población de alto riesgo a través de la diferencia en unidades al multiplicar el porcentaje de la población por un factor de riesgo por el cociente de peligro, menos la diferencia del sector poblacional de no riesgo. Lo cual se interpreta como una mayor probabilidad de exposición. Y se calcula con la siguiente fórmula:

$$
P E=\left(\frac{(S P R)(F R)}{(S P)(F R)}\right)(C P)
$$

Dónde: (PE) Probabilidad de exposición; (SPR) Sector poblacional de riesgo (conformado por niños de 0-12 años y adultos mayores >60 años); (SP) Sector poblacional no riesgo (conformado por adolescentes de 13-18 años y adultos de 19 a 59 años); (FR) se asume (1: Por ser miembro de la población; 2: por pertenecer a población vulnerable); $(\mathrm{CP})$ Cociente de peligro (ver indicador I de peligro).

a) Probabilidad de exposición alta $(\mathrm{PE} \geq 20)=5$

b) Probabilidad de exposición media. (PE 10-19) $=4$

c) Probabilidad de exposición baja $(\mathrm{PE} \leq 10)=3$

d) Probabilidad de exposición desconocida $=1$

3) Probabilidad de exposición basada en su permanencia ambiental y su relación con el periodo de vida del sector expuesto: Este rubro se refiere al periodo de tiempo promedio que vive el sector expuesto en condiciones naturales, a mayor permanencia de un contaminante en el ambiente mayor será su probabilidad de exposición para el sector expuesto (MINAM, 2015).

Tabla 2. Tipos de exposición (adaptado de Peña et al., 2001 y

\begin{tabular}{|c|c|c|}
\hline $\begin{array}{c}\text { Tipo de } \\
\text { exposición }\end{array}$ & Lapso de exposición & Definición \\
\hline Duradera & $\begin{array}{c}\text { Entre } 10 \% \text { y el } \\
100 \% \text { del periodo de } \\
\text { vida (en humanos de } \\
7 \text { a } 70 \text { años). }\end{array}$ & $\begin{array}{l}\text { Contacto perdurable a lo } \\
\text { largo de un periodo de } \\
\text { vida del organismo }\end{array}$ \\
\hline Ocasional & $\begin{array}{c}<10 \% \text { del periodo } \\
\text { de vida (según la } \\
\text { edad actual) }\end{array}$ & $\begin{array}{c}\text { Contacto directo de } \\
\text { manera frecuente pero } \\
\text { poco alargada del } \\
\text { organismo en la matriz }\end{array}$ \\
\hline Accidental & $\begin{array}{c}<1 \text { día como único } \\
\text { evento }\end{array}$ & $\begin{array}{l}\text { Contacto fortuito del } \\
\text { organismo con la matriz }\end{array}$ \\
\hline
\end{tabular}
Quiterio-Pérez, 2012). a) Probabilidad de exposición alta. Exposición duradera $=5$

b) Probabilidad de exposición media. Exposición ocasional $=4$

c) Probabilidad de exposición baja. Exposición accidental $=3$

d) Probabilidad de exposición desconocida $=1$

4) Exposición efectiva basada en su biodisponibilidad vs factores ambientales: Se refiere a la posibilidad que ejercen los factores ambientales (pH y temperatura) (Vicente-Martonell et al., 2009) para favorecer la biodisponibilidad (entendiéndose esta, como la capacidad que tienen los compuestos xenobióticos de ingresar a sistemas biológicos).

a) Biodisponibilidad alta ( $\mathrm{pH}$ por debajo de $7 \mathrm{y}$ temperaturas por encima de $\operatorname{los} 25^{\circ} \mathrm{C}$ ) $=5$

b) Biodisponibilidad media alta $(\mathrm{pH}$ por debajo de 7 y temperaturas por debajo de $\left.\operatorname{los} 25^{\circ} \mathrm{C}\right)=4$

c) Biodisponibilidad media baja $(\mathrm{pH}$ por encima de $7 \mathrm{y}$ temperaturas por encima de $\left.\operatorname{los} 25^{\circ} \mathrm{C}\right)=3$

d) Biodisponibilidad baja ( $\mathrm{pH}$ por encima de $7 \mathrm{y}$ temperaturas por encima de $\left.\operatorname{los} 25^{\circ} \mathrm{C}\right)=2$

e) Biodisponibilidad desconocida $=1$

5) Exposición efectiva basada en lipofilicidad: Este rubro se refiere a la capacidad que posee un compuesto xenobiótico de unirse a compuestos lipídicos, Esta capacidad dependerá de la constante de equilibrio (pKs) del compuesto xenobiótico (GarritzRuiz et al., 2005). Un incremento progresivo en el valor de pKs representa una mayor lipofilicidad y, por lo tanto, la posibilidad para atravesar membranas lipídicas y posibilidad de interacción molecular aumenta (Roldan, 2016).

a) Valor de $\mathrm{pKs}(\geq 20)$. Altamente lipófilo $=5$

b) Valor de pKs (10-19). Medianamente lipófilo = 4

c) Valor de $\mathrm{pKs}(\leq 9)$. Baja lipofília $=3$

d) Valor de $\mathrm{pKs}$ desconocido $=1$

$>$ Evaluación dosis-respuesta a través del efecto biológico: La evaluación dosis-respuesta pretende obtener una estimación de la proporción entre la cantidad de los compuestos xenobióticos a la cual están expuestos el sector poblacional y la incidencia o severidad de una respuesta o efecto (OMS, 2017); dichos efectos pueden ser determinados a través de 5 indicadores: cinética de eliminación, factor de Bioconcentración, potencial de toxicidad, potencial de efecto teratogénico y riesgo cancerígeno.

1) Efecto por cinética de eliminación basada en vida media: Este rubro se refiere a la velocidad con la que los compuestos xenobióticos se eliminan del organismo. La cinética de eliminación se expresa mediante la constante de eliminación y la vida media biológica. Cuanto más rápida sea la eliminación del compuesto, menor será la posibilidad de permanecer dentro del organismo y ocasionar efectos adversos (Armijo, 2014). Y se calcula mediante la siguiente fórmula:

$$
T_{1 / 2}=\ln 2 / K_{e}
$$

Dónde: $\left(T_{1 / 2}\right)$ corresponde al tiempo que tarda la concentración del compuesto en reducirse a la mitad; $(\ln 2) \operatorname{logaritmo}$ natural de 2 ; $K_{e}$ indica la probabilidad de eliminación expresado en días.
a) Efecto adverso alto, vida media $\left(\mathrm{T}_{1 / 2} \geq 10^{-6}\right)=5$
b) Efecto adverso medio-alto, vida media $\left(\mathrm{T}_{1 / 2} 10^{-4}\right.$ a $\left.10^{-5}\right)=4$
c) Efecto adverso medio, vida media $\left(\mathrm{T}_{1 / 2} 10^{-1}\right.$ a $\left.10^{-3}\right)=3$
d) Efecto adverso bajo, vida media $\left(T_{1 / 2} \leq 10^{-1}\right)=2$
e) Efecto adverso desconocido $=1$

2) Efecto por factor de bioconcentración $(F B C)$ : Este rubro se refiere a la relación entre la acumulación del metal en el medio 
(agua) y la biomasa del organismo (Yadav et al., 2018). De acuerdo con Ali et al. (2013), donde si el valor de FBC es mayor a $1(\mathrm{FBC}>1)$, indica una alta capacidad de bioacumulación del contaminante en el organismo $\mathrm{y}$, por lo tanto, aumenta su posibilidad de daño.

$$
F B C=\frac{C_{o}}{C_{m}}
$$

Dónde: $(F B C)$ Factor de Bioconcentración; $\left(C_{o}\right)$ concentración del metal en el organismo (ATSDR, 2005); $\left(C_{m}\right)$ concentración del metal en el medio a evaluar (concentración reportada).

a) Alta bioacumulación $(\mathrm{FBC} \geq 1)=5$

b) Mediana bioacumulación $(\mathrm{FBC}=1)=4$

c) Baja bioacumulación $(\mathrm{FBC} \leq 1)=3$

d) FBC Desconocido $=1$

3) Efecto por potencial de toxicidad basado en $D L_{50}$ : Este rubro se refiere a la posibilidad de que una sustancia ocasione un efecto biológico basado en el potencial de toxicidad y el peso molecular de la misma. De acuerdo al criterio de Degrossi (2013), compuestos con un potencial de toxicidad (pT) alto pueden ocasionar efectos adversos en los organismos. Y se calcula mediante la siguiente fórmula:

$$
p T=-\log T
$$

Dónde: (T) Dosis toxica molar en mol/kg, se calcula a partir de la $\mathrm{DL}_{50}$ oral en ratas.

$$
T=\frac{D L_{50} / 1000}{\text { Peso molecular }}
$$

a) Potencial de toxicidad alta $(>10)=5$

b) Potencial de toxicidad media $(5-10)=4$

c) Potencial de toxicidad baja $(1-5)=3$

d) Potencial de toxicidad desconocida $=1$

4) Probabilidad de efecto biológico basado en su Potencial de Efecto teratogénico (PET): Este rubro se refiere a la capacidad potencial de los compuestos xenobióticos de inducir malformaciones o defectos en la descendencia, basado en reportes científicos y estudios clínicos del incremento de la frecuencia de malformaciones estructurales, congénitas, no-hereditarias en la progenie y visualmente detectables al nacimiento, que pueden ser asociadas a la exposición a compuestos xenobióticos durante el desarrollo embrionario (Repetto-Jimenez y Sanz, 1995).

a) Potencial teratogénico probado. Se reportan efectos de daño durante el desarrollo embrionario en estudios clínicos en humanos $=5$

b) Potencial teratogénico probable. Se reportan efectos positivos en animales mamíferos durante el desarrollo embrionario y los estudios clínicos en humanos son escasos $=4$

c) Potencial teratogénico incierto. Se reportan efectos de daño durante el desarrollo embrionario en animales no mamíferos, los estudios clínicos en humanos no existen $=3$

d) Potencial teratogénico desconocido $=1$

5) Efecto por riesgo cancerígeno: Este rubro se refiere a la posibilidad de desarrollo de cáncer por exposición a un compuesto xenobiótico (USEPA, 2001). El riesgo de desarrollo de cáncer durante el tiempo de visa asociado a la exposición se calcula a partir de datos de la ingesta crónica diaria (CDI) y el factor de pendiente (SF) establecido en la base de datos IRIS de la EPA (2005) o de la ATSDR (2004) para cada compuesto xenobiotico. La ingesta crónica diaria (CDI), se calculó mediante la siguiente fórmula:

$$
C D I=\frac{(C)(T I)}{B W}
$$

Dónde: (CDI) ingesta crónica diaria (mg/kg/día); (C) concentración del contaminante en agua (reportada) $(\mathrm{mg} / \mathrm{L})$; (TI) tasa de ingesta diaria de consumo de agua potable (L/d) 2 litros/adulto, 1.5 litros/niño.

$$
L C R i=[C D I(m g / k g / d)] x[S F(m g / k g / d)]
$$

Dónde: LCRi: Riesgo de desarrollo de cáncer individual durante el tiempo de vida; $C D I$ : es la ingesta crónica diaria $(\mathrm{mg} / \mathrm{kg} / \mathrm{d}) ; S F$ : es el factor de pendiente establecido en la base de datos IRIS de la EPA (2005) o de la ATSDR (2004) por contaminante expresado en $(\mathrm{mg} / \mathrm{kg} / \mathrm{día})$.

Para estimar el riesgo cancerígeno poblacional $\left(L C R_{p}\right)$ se multiplica el riesgo cancerígeno individual $\left(L C R_{i}\right)$ por el número total de la población para de este modo obtener el riesgo cancerígeno poblacional; el valor obtenido representa un escenario hipotético que debe interpretarse como el número de casos de cáncer que aparecerían en una población (USEPA, 2001; CEPIS/OPS, 2005).

$$
L C R p=[L C R i(\mathrm{mg} / \mathrm{kg} / \mathrm{d})] x P T
$$

Dónde: LCRp: Riesgo de desarrollo de cáncer poblacional durante el tiempo de vida; LCRi: Riesgo de desarrollo de cáncer individual durante el tiempo de vida; PT: Población total (40, 201 habitantes).

a) Probabilidad alta de incidencia de cáncer $(\geq 30)=5$

b) Probabilidad media de incidencia de cáncer $(11-20)=4$

c) Probabilidad baja de incidencia de cáncer $(0-10)=3$

d) Probabilidad de incidencia de cáncer desconocida $=1$

\section{Resultados}

De acuerdo a los resultados obtenidos en el análisis de causa y efecto, se establecieron 15 indicadores con respuesta múltiple, con valores de respuesta de 1 a 5; en la tabla 3, se muestra la matriz de dos vías con las variables correspondientes a los indicadores imperativos de Peligro, Exposición y Efecto. Dentro de la matriz los valores que oscilan entre 1 y 5 se identificaron colorimétricamente de acuerdo al nivel de riesgo como se observa en la figura 2 de escala de puntuación. En la suma total de los valores por cada uno de los compuestos, la puntuación total para cromo $(\mathrm{Cr})$ y cadmio $(\mathrm{Cd})$ fue de 66, arsénico (As) de 65 y plomo $\mathrm{Pb})$ de 58, ubicándolos dentro de la categoría de Potencial de Riesgo Alto identificado con el color rojo (Figura 2); mientras que para mercurio $(\mathrm{Hg})$ se presenta valor de 56 puntos respectivamente, ubicándolos en la categoría de Potencial de Riesgo Intermedio.

\begin{tabular}{|c|c|c|}
\hline Valor & & Probabilidad \\
\hline 5 & & Alta \\
\hline 4 & & Media-alta \\
\hline 3 & & Media \\
\hline 2 & & Baja \\
\hline 1 & & Incertidumbre \\
\hline
\end{tabular}

\begin{tabular}{|c|c|}
\hline Categoría & Puntuación \\
\hline & Potencial de riesgo alto 68-90 \\
\hline & Potencial de riesgo intermedio 45-67 \\
\hline & Potencial de riesgo bajo 22-44 \\
\hline & Potencial de riesgo nulo $<21$ \\
\hline
\end{tabular}

Figura 2. Escala de puntuación de sumatoria final y por indicador (Gaytán-Oyarzun et al., 2020). 
Tabla 2. Matriz de dos vías con variables y valores obtenidos.

\begin{tabular}{|c|c|c|c|c|c|c|}
\hline \multirow{2}{*}{\multicolumn{2}{|c|}{ Indicador }} & \multicolumn{5}{|c|}{ Metales } \\
\hline & & \multirow{2}{*}{$\begin{array}{c}\mathbf{A s} \\
5\end{array}$} & \multirow{2}{*}{$\frac{\mathbf{C r}}{4}$} & \multirow{2}{*}{$\begin{array}{c}\mathbf{C d} \\
5\end{array}$} & \multirow{2}{*}{$\frac{\mathbf{P b}}{5}$} & \multirow{2}{*}{$\frac{\mathbf{H g}}{4}$} \\
\hline \multirow{5}{*}{$\begin{array}{l}\mathbf{P} \\
\mathbf{E} \\
\mathbf{L} \\
\mathbf{I} \\
\mathbf{G} \\
\mathbf{R} \\
\mathbf{O}\end{array}$} & I) Concentración & & & & & \\
\hline & II) EMEG & 5 & 5 & 5 & 1 & 3 \\
\hline & III) Presencia Múltiple & 5 & 5 & 5 & 5 & 5 \\
\hline & IV) Persistencia & 2 & 5 & 5 & 5 & 3 \\
\hline & V) Solubilidad en agua & 4 & 4 & 4 & 4 & 4 \\
\hline \multirow{5}{*}{$\begin{array}{c}\mathbf{E} \\
\mathbf{X} \\
\mathbf{P} \\
\mathbf{O} \\
\mathbf{S} \\
\mathbf{I} \\
\mathbf{C} \\
\mathbf{I} \\
\mathbf{O} \\
\mathbf{N}\end{array}$} & I) Tasa de exposición & 5 & 5 & 4 & 4 & 3 \\
\hline & II) PESP & 5 & 4 & 3 & 3 & 3 \\
\hline & III) PASE & 5 & 5 & 5 & 5 & 5 \\
\hline & IV) Biodisponibilidad & 5 & 5 & 4 & 4 & 4 \\
\hline & V) Lipofilicidad & 4 & 4 & 4 & 4 & 4 \\
\hline \multirow{5}{*}{$\begin{array}{l}\mathbf{E} \\
\mathbf{F} \\
\mathbf{E} \\
\mathbf{C} \\
\mathbf{T} \\
\mathbf{O}\end{array}$} & I) Cinética de eliminación & 2 & 5 & 5 & 5 & 4 \\
\hline & II) $\mathrm{FBC}$ & 5 & 3 & 5 & 3 & 3 \\
\hline & III) $\mathrm{PT}$ en $\mathrm{DL}_{50}$ & 3 & 3 & 3 & 3 & 3 \\
\hline & IV) $\mathrm{pET}$ & 5 & 4 & 5 & 3 & 5 \\
\hline & V) Riesgo cancerígeno & 5 & 5 & 4 & 4 & 3 \\
\hline & Total & 65 & 66 & 66 & 58 & 56 \\
\hline
\end{tabular}

(EMEG) Concentración ambiental estimada; (PESP) Probabilidad de exposición en el sector poblacional; (PASE) Permanencia ambiental en el sector poblacional; (FBC) Factor de Bioconcentración; (PT) Potencial de toxicidad: (DL ${ }_{50}$ ) Dosis letal media; (pET) Potencial de efecto teratogénico.

\section{Discusión}

El método de Evaluación Rápida de Potenciales de Riesgo (ERPRG), es una metodología rápida, clara y eficaz que apoya la toma de decisiones, además es una herramienta que permite identificar situaciones de potencial de riesgo biológico $\mathrm{y}$ ambiental, basada en el análisis sistemático de reportes bibliográficos, estudios previos y observaciones simples para determinar cualitativamente el impacto de variables ambientales, biológicas y situacionales. Autores como Lee-Steere (2009) mencionan que en la evaluación de riesgos no necesariamente se requiere de la aplicación de técnicas sofisticadas o la recolección excesiva de datos. Para ello se pueden obtener resultados prácticos y razonables utilizando información mínima disponible sobre la contaminación y la población expuesta. De igual manera, en el presente estudio, la información recabada para la ejecución de la técnica cumple con ciertos requisitos para ser considerada al momento de la consulta, además de no requerir fases experimentales para obtener información.

Como parte de los métodos de evaluación rápida, el método ERPRG confirma su eficacia con la capacidad de identificar el potencial de riesgo de compuestos xenobióticos, previo a análisis químicos y de efecto biológico a través de información ya descrita basada en cinética ambiental, cuestiones ambiente situacionales, exposición, biodisponibilidad, sectores expuestos, efectos potenciales y reportados. Tiene la versatilidad de adaptarse a alguna cuestión en particular que requiera ser analizada; por ello, es considerada como una nueva metodología que puede ser modificada y actualizada en la determinación del potencial de riesgo de diferentes compuestos xenobióticos. Es así, como se han empleado los métodos de evaluación rápida con diferentes propósitos, con respectivas adecuaciones en cuanto a la identificación de variables y la ejecución de las interrogantes del análisis de causa y efecto, que proporcionen información necesaria en su implementación, así como las adaptaciones realizadas en la Evaluación Rápida de Biodiversidad para Estimar Prioridad Biológica (ERBio) (Gaytán et al., 2012); Evaluación Rápida de Potenciales de Riesgo (ERPR) (Quiterio-Pérez, 2012; Gaytán y López, 2019); Evaluación de Potencial de Riesgos Ambientales y Efectos a la Salud (EPRAS) Rodríguez-Anaya et al., 2014); Evaluación Rápida de Potencial de Riesgo Genotóxico (ERPOR-G) (Gaytán-Oyarzun et al., 2020)

En el sitio de estudio correspondiente al municipio de Zimapán, Hidalgo, se cuenta con reportes de concentraciones altas de arsénico (Armienta et al., 2001; Pérez-Moreno, 2004), el cual se encuentra presente de manera natural en el ambiente debido a su origen geológico, gran parte de los estudios realizados se encuentran enfocados a los efectos que este metaloide ocasiona en los organismos. Sin embargo, existe evidencia de la presencia de otros metales pesados reportados en el sitio de estudio como el plomo, cadmio, cromo y mercurio, con características fisicoquímicas y toxicológicas que requieren ser estudiados desde un enfoque ecotoxicológico por su riesgo potencial.

En los resultados de la aplicación del método ERPRG, se demostró que, de los metales pesados de interés toxicológico identificados en el sitio de estudio, además del arsénico (As), metaloide clasificado en la categoría de potencial de riesgo alto derivado de la presente investigación; se encuentran metales pesados como el cromo $(\mathrm{Cr})$, cadmio $(\mathrm{Cd})$ y plomo $(\mathrm{Pb})$ ubicados en la misma categoría de riesgo; mientras que el mercurio $(\mathrm{Hg})$ se ubica en la categoría de riesgo intermedio. Esto pone de manifiesto la necesidad de estudiar estos metales con particular atención, ya que por sus características se ubican en categorías de riesgos con la probabilidad de ocasionar efectos adversos tanto en el ambiente, como en la biota e incluso el ser humano. Ya que, inicialmente, la concentración de un compuesto o sustancia presente en el ambiente resultaba ser suficiente para llevar a cabo acciones para regular su presencia. Sin embargo, actualmente variables como la permanencia en el ambiente del compuesto, biodisponibilidad, potencial de toxicidad y los efectos biológicos potenciales de exposición resultan ser relevantes para llevar a cabo acciones de regulación y la formulación de normativas, y de este modo ampliar la investigación acerca de los principales metales de interés toxicológico que se encuentran presentes en el ambiente.

\section{Conclusiones}

El método de Evaluación Rápida de Potencial de Riesgo Genotóxico (ERPRG) permite la toma decisiones rápidas y prioriza aquellos contaminantes que requieren ser estudiados con un enfoque ecotoxicológico y se centra en el análisis de las interrelaciones de las variables ambientales, de los sectores expuestos y propias de los compuestos químicos a evaluar, permitiendo identificar ambientes peligrosos, posibles sectores expuestos de mayor riesgo y efectos potenciales.

Y para el caso particular de Zimapán, este método clasifica a dos metales pesados y un metaloide con un potencial de riesgo alto (arsénico, cromo y cadmio); plomo y mercurio con un potencial de riesgo intermedio, lo que indica que cada uno de ellos requiere ser estudiado con particular atención desde un enfoque toxicológico. 


\section{Agradecimientos}

Los autores agradecen a la Universidad Autónoma del Estado de Hidalgo, por las facilidades institucionales en la realización de la presente investigación. MASO agradece al CONACYT por la beca doctoral en el programa de Doctorado en Ciencias Ambientales.

\section{Referencias}

Agency for Toxic Substances and Disease Registry (ATSDR)., (2004). Public Health Assessment. Guidance Manual (Update). Atlanta, Georgia: U. S. Department of Health and Human Services; [acceso 2018 Dic 15]. https://www.atsdr.cdc.gov/hac/phamanual/toc.html

Agency for Toxic Substances and Disease Registry (ATSDR)., (2005). Public Health Assessment. Guidance Manual (Update). Atlanta, Georgia: U. S. Department of Health and Human Services; [acceso 2020 Ago 19]. https://www.atsdr.cdc.gov/hac/phamanual/toc.html

Ali, H., Khan, E., y Anwar, M., (2013). Phytoremediation of heavy metalsConcepts and application. Chemosphere 7(91), 869-881. DOI: 10.1016/j.chemosphere.2013.01.075.

Armienta MA, Villaseñor G, Rodríguez R, Ongley LK, Mango H. (2001). The role of arsenic-bearing rocks in groundwater pollution at Zimapán Valley, México. Environmental Geology 40, 571-581. DOI: $10.1007 / \mathrm{s} 002540000220$

Armijo, S. J. A., (2014). Farmacocinética: absorción, distribución y eliminación de los fármacos. En: Farmacología humana. 6a . Edición. Elsevier Ed. Jesús Florez. España.

Centro Panamericano de Ingeniería Sanitaria y Ciencias del ambiente/Organización Panamericana de la Salud (CEPIS/OPS), (2005). Curso de autoinstrucción. Obtenido de Evaluación de Riesgo asociado a Contaminantes de Aire. [acceso 2020 Apr 13]. http://www.bvsde.paho.org/cursoa_riesgoaire/lecciones/leccion2d.html

Chambers, R., (1983). Rural development: putting the last first. Harlow: Prentice Hall.

Combariza, B. D. A., (2009). Contaminación por metales pesados en el embalse del Muña y su relación con los niveles en sangre de plomo, mercurio y cadmio y su alteración de salud en los habitantes del municipio de Sibaté (Cundinamarca) [Tesis de Maestría]. Colombia: Universidad Nacional de Colombia.

Degrossi, M. C., (2013). Toxicología de alimentos. En Universidad de Belgrano. Argentina: Ed. Facultad de Ciencias de la Salud.

Diario Oficial de la Federación (DOF)., (2017). PROY-NOM-127-SSA1-2017. Agua para uso y consumo humano. Límites permisibles de la calidad del agua. México. [acceso $2019 \quad$ Oct https://www.dof.gob.mx/nota_detalle.php?codigo $=5581179 \&$ fecha $=06 / 12 /$ 2019

Díaz-Barriga, F., (1999). Metodología de identificación y evaluación de riesgos para la salud en sitios contaminados. Perú: Ed. Centro Panamericano de Ingeniería Sanitaria (OPS)/ Organización Panamericana de Salud.

Environmental Protection Agency (EPA)., (2005). Integrated Risk Information System (IRIS). Washington, D.C. U. S. [acceso 2019 Sep 19]. https://cfpub.epa.gov/ncea/iris_drafts/atoz.cfm?list_type=alpha

Garritz-Ruiz, A., Gasque-Silva, L., Martínez-Vázquez, A., (2005). Equilibrios de solubilidad. En: Química Universitaria. Pearson Educación, México, pp. 609-621.

Gaytán-Oyarzun, J. C., López-Escamilla, A., López-Herrera, M., PulidoFlores, G., Monks, S. W., Pérez-Escandón, B., Villavicencio-Nieto, M., (2012). Evaluación rápida de biodiversidad para estimar prioridad biológica (ERPBIO). En Sampedro-Rosas ML, y González-González J. Calidad Ambiental y Desarrollo Sustentable: Indicadores. Tomo II. México DF. Ed. Universidad Autónoma de Guerrero e INDAUTOR.

Gaytán-Oyarzun, J. C., y López, H. M., (2019). Plaguicidas, uso y potencial de riesgo. En: Sustentabilidad y Gestión Ambiental. UAZ "Francisco García Salinas" pp. 309.

Gaytán-Oyarzun, J. C., Sánchez-Olivares, M. A., Cabrera-Cruz. R. B. E., (2020). Evaluación potencial de riesgo genotóxico de compuestos xenobióticos. En: Pichardo-Ramírez, R., Tobías-Jaramillo, R., y J. TreviñoTrujillo. Investigaciones actuales para el medio ambiente II. Universidad Autónoma de Tamaulipas. México, pp 85-99.

González-Mille, D. J., Ilizaliturri-Hernández, C. A., Espinosa-Reyes, G., Costilla-Salazar, R., Díaz-Barriga, F., Ize-Lema, I., Mejía-Saavedra, J., (2010). Exposure to persistent organic pollutants (POPs) and DNA damage as an indicator of environmental stress in fish or different feeding habits of Coatzacoalcos, Veracruz, México. Ecotoxicology. 19, 1238-1248.

Lee-Stere, C., (2009). Environmental risk assessment guidance manual for industrial chemicals. Australia: Environment Protection and Heritage Council.

Liu, X., Wu, J., Xu, J., (2006). Characterizing the Risk assessment of heavy metals and sampling uncertainty analysis in paddy field by geostaticks and GIS. Environ Pollut. 141(2), 257-254. DOI: 10.1016/j.envpol.2005.08.048

Martín-Olmedo, P., Carroquino-Saltó, M.J., Ordoñez-Iriarte, J.M., y J. Moya., (2016). La evaluación de riesgos en salud. Guía metodológica. Aplicaciones prácticas de la metodología de la evaluación de riesgos en salud por exposición a químicos. 3er ed. Ed. Sociedad Española de Sanidad Ambiental (SESA) y Escuela Andaluza de Salud Pública, Madrid. 260 p.

Ministerio del Ambiente (MINAM)., (2015). Guía para la elaboración de estudios de Evaluación de Riesgos a la Salud y el Ambiente (ERSA) en sitios contaminados. Ed. Ministerio del Ambiente, Dirección general de calidad ambiental, Lima, Perú. 158 p, consultado: http://www.minam.gob.pe/calidadambiental/wpcontent/uploads/sites/22/20 15/02/GUIA-ERSA-ALTA.compressed.pdf.

Nava-Ruíz, C., Méndez-Armenta, M., (2011). Efectos neurotóxicos de metales pesados (cadmio, plomo, arsénico y talio). Arch Neurocien. 16(3), 140-147.

Organización Mundial de la Salud (OMS)., (2017). Herramienta de evaluación de riesgos para la salud humana de la OMS: peligros quimios. Ed. Organización Mundial de la Salud (OMS). Documento n.8 del Proyecto de armonización del IPCS. Ginebra, $110 \mathrm{pp}$

Peña, C.E., Carter, E.D., y F. Ayala-Fierro., (2001). Toxicología Ambiental: Evaluación de Riesgos y Restauración Ambiental. En the Southwest Hazardous Waste Program, Universidad de Arizona. 204 p, consultado: http://www.ingenieroambiental.com/informes2/toxamb.pdf.

Pérez-Moreno, F., (2004). Dinámica del arsénico en aguas subterráneas de pozos y sedimentos del distribuidor general de agua potable de Zimapán, Hidalgo. [Tesis de Doctorado]. Pachuca, Hidalgo, México: Centro de Investigaciones Químicas. Universidad Autónoma del Estado de Hidalgo. Pachuca Hidalgo. 2004

Pérez-Rodrigo, C., Morán-Fagúndez, L. J., Riobó-Servan, P., Aracenta, J., (2015). Métodos de cribado y métodos de evaluación rápida. Rev Esp Nutr Comunitaria. 21(1), 88-95. DOI: 10.14642/RENC.2015.21.sup1.5056.

Quiterio-Pérez, M., (2012). Evaluación espacio temporal de pesticidas organoclorados (POs) en aguas y sedimentos de la laguna de Metztitlán, Reserva de la Biosfera de la Barranca de Metztitlán, Hidalgo. Tesis para obtener el grado de Doctor en Ciencias Ambientales, UAEH, México.

Ramírez, J. A., Lacasaña, M., (2001). Plaguicidas: clasificación, uso, toxicología y medición de la exposición. Arch Prev Riesgos Labor. 4(2), 6775.

Repetto-Jiménez, M., y P. Sanz., (1995). Glosarios de términos toxicológicos IUPAC (Duffus y cols. 1993). En Asociación Española de Toxicología, consultado: https://www.aetox.es/glosario-toxicologico.

Rodríguez-Anaya, A., Gaytán-Oyarzun, J. C., Gordillo-Martínez, J. A., PrietoGarcía, F., Marmolejo-Santillán, Y., (2014). Model for estimating the environmental risk potential per presence of drugs nonsteroidal antiinflammatory (NSAIDS) consumption high. Ciencia e Técnica Vitivinícola $29,51-76$.

Roldan, R. E., (2016). Introducción a la toxicología. Ed. UNAM, FES Zaragoza. $141 \mathrm{pp}$

Tambutti, M., Aldama, A., Sánchez, O., Medellin, R., Soberón, J., (2001). La determinación del riesgo de extinción de especies silvestres en México. Gaceta Ecológica. 61, 11-21.

U. S. Environmental Protection Agency (USEPA). (2001). United States Environmental Protection Agency. Guidelines for Carcinogen Risk Assessment, Risk Assessment Forum; Washington D.C.; United States of America.

Vicente-Martonell, J. J., Galindo-Riaño, M. D., García-Vargas, M., GranadoCastro, M. D., (2009). Bioavailability of heavy metals monitoring water, sediments and fish species from a polluted estuary. Journal of Hazardous Materials. 162(2-3): 823-836. DOI: 10.1016/j.jhazmat.2008.05.106.

World Health Organization (WHO)., (1982). Rapid assessment of sources of air, water, and land pollution. World Health Organization. Geneva, Switzerland

Yadav, K. K., Gupta, N., Kumar, A., Reece, L. M., Singh, N., Rezania, S., y Khan, S. A., (2018). Mechanistic understanding and holistic approach of phytoremediation: A review on application and future prospects. Ecological Engineering. 120, 274-298. DOI: 10.1016/j.ecoleng.2018.05.039

Zepeda, C. H., Gómez-Tagle, R. A. F., Chávez, H. Y., (2002). Metodología rápida para la evaluación de ecosistemas riparios en zonas templadas. Ing Hidráulica en México. 13(1), 61-74. 